\title{
Estimativa dos Impactos Ambientais e Socioeconômicos dos Últimos 3 Grandes Eventos de Seca $(2005,2010$ e 2015) na Amazônia
}

\author{
Waldeir dos Santos Pereira ${ }^{1}$, Gabriel Brito Costa ${ }^{1}$, Duany Thainara Corrêa da Silva ${ }^{1}$, \\ Letícia Victória dos Santos Matias ${ }^{1}$, Ana Caroline da Silva Macambira ${ }^{1}$, \\ Marcelo de Almeida Heidemann ${ }^{1}$, Natan Barbosa Almada ${ }^{1}$ \& Adailton Silva Santos ${ }^{1}$
}

Recebido em 15/09/2020 - Aceito em 02/07/2021 1 Universidade Federal do Oeste do Pará/UFOPA. Brasil. <waldeir.santtos@hotmail.com, gabrielbritocosta@gmail.com,
duanythaynara@gmail.com, lleticia005@gmail.com, karoline_macambira@hotmail.com, marceloaheidemann@hotmail.com,
natanalmada@gmail.com, adailtonfisica10@gmail.com>.

RESUMO - O objetivo deste trabalho foi analisar os impactos das secas ocorridas nos anos de 2005, 2010 e 2015, na região Amazônica, quanto a fatores ambientais (temperatura do ar, umidade relativa, risco de incêndios florestais) e socioeconômicos (consumo de energia elétrica). Foram analisados dados de temperatura e umidade relativa do ar de 41 estações meteorológicas de um período de 38 anos estimando os riscos de incêndios florestais (pela Fórmula de Monte Alegre), e dados estaduais de consumo de energia elétrica, distintos por tipo de consumo (residencial, industrial, comercial ou rural) e média anual registrada. Os resultados mostraram que as secas em análise, embora tenham gêneses diferentes (2005 devido às anomalias no atlântico, 2010 e 2015 devido aos fortes El Niños), tiveram fortes impactos ambientais (médias diárias de temperatura do ar acima de $32^{\circ} \mathrm{C}$, valores de umidade relativa do ar próximos de $20 \%$, fator de risco de ocorrência de incêndios florestais muito alto) e socioeconômicos (aumento no consumo de energia elétrica em até 200GWh nos anos de seca, principalmente no modo residencial), indicando que anos com previsão de ocorrência de secas severas como os estudados neste trabalho precisam do preparo do poder público quanto aos efeitos da elevação das temperaturas e do consumo energético, necessitando medidas profiláticas quanto aos impactos negativos gerados.

Palavras-chave: Clima; eventos extremos; precipitação.

\section{Environmental and Socioeconomic Estimated Impacts of the Last 3 Major Drought Events (2005, 2010 and 2015) in the Amazon}

\begin{abstract}
The objective of this work was to analyze the impacts of droughts that occurred in the years of 2005, 2010 and 2015 in the Amazon region, regarding environmental factors (air temperature, relative humidity, risk of forest fires) and socioeconomic factors (consumption of electricity). Data on temperature and relative humidity of 41 weather stations over a period of 38 years were analyzed, estimating the risks of forest fires (using the Monte Alegre formula), and state electricity consumption data, different by type of consumption (residential, industrial, commercial or rural) and annual average recorded. The results showed that the droughts focused, although having different genesis (2005 due to anomalies in the Atlantic, 2010 and 2015 due to strong El Niños) had strong environmental impacts showed strong environmental impacts (daily air temperature averages above $32^{\circ} \mathrm{C}$, relative humidity values near to $20 \%$, fire danger for the occurrence of forest fires high) and socioeconomic (increase in electricity consumption by up to 200GWh in dry years, mainly in residential mode), indicating that years with prediction of severe droughts such as those studied in this work need the preparation of the public authorities regarding the effects of rising temperatures and energy consumption, requiring prophylactic measures regarding the negative impacts generated.
\end{abstract}

Keywords: Climate; extreme events; rainfall.

\section{Impactos Ambientales y Socioeconómicos Estimados de los Últimos 3 Grandes Eventos de Sequía (2005, 2010 y 2015) en la Amazonía}

RESUMEN - El objetivo de este trabajo fue analizar los impactos de las sequías ocurridas en los años 2005, 2010 y 2015 en la región Amazónica, en cuanto a factores ambientales (temperatura 


\begin{abstract}
del aire, humedad relativa, riesgo de incendios forestales) y socioeconómicos (consumo de energía eléctrica). Se analizaron datos de temperatura y humedad relativa de 41 estaciones meteorológicas en un período de 38 años, estimando los riesgos de incendios forestales (utilizando la fórmula de Monte Alegre), y datos de consumo eléctrico estatal, diferentes por tipo de consumo (residencial, industrial, comercial). o rural) y promedio anual registrado. Los resultados mostraron que las sequías focalizadas, aunque con diferente génesis (2005 por anomalías en el Atlántico, 2010 y 2015 por fuertes El Niño) tuvieron fuertes impactos ambientales mostraron fuertes impactos ambientales (promedios diarios de temperatura del aire por encima de $32^{\circ} \mathrm{C}$, humedad relativa valores cercanos al $20 \%$, peligro de incendio por ocurrencia de incendios forestales alto) y socioeconómico (aumento del consumo eléctrico hasta en 200GWh en años secos, principalmente en modo residencial), indicando que años con predicción de sequías severas como las estudiadas En este trabajo es necesaria la preparación de las autoridades públicas sobre los efectos del aumento de temperaturas y el consumo de energía, requiriendo medidas profilácticas frente a los impactos negativos generados.
\end{abstract}

Palabras clave: Clima; eventos extremos; lluvia.

\section{Introdução}

A Amazônia tem importância no cenário mundial por conter uma ampla área de floresta tropical (Pereira, 2006), obtendo, assim, grande influência no clima do planeta, que, segundo Parry (2007) e Jon (2009), está sofrendo alterações devido à emissão de gases principalmente com o efeito estufa, o que influencia no aumento da sua temperatura e o expõe a um maior número de fenômenos meteorológicos a citar as alterações nos índices pluviométricos. Segundo o Painel Intergovernamental sobre Mudanças Climáticas (IPCC, 2018), restringir a um padrão de $1,5^{\circ} \mathrm{C}$ o limite médio de aumento de temperatura global é uma meta importante e desafiadora para amenizar futuramente impactos climáticos severos.

No Brasil, as duas regiões que estarão mais suscetíveis a possíveis eventos extremos são o Nordeste e a Amazônia (Marengo, 2007; Ambrizzi et al., 2007; Marengo et al., 2007). Cientistas estimam uma possível alteração da floresta Amazônica (Galbraith et al., 2010; Good et al. 2011; Settele et al., 2014), onde, aproximadamente, $60 \%$ de sua área poderá ser modificada (Oyama \& Nobre, 2003), fato este que favorecerá o aumento dos riscos de incêndios e queimadas na região devido às alterações climatológicas e eventos de seca extrema que poderão transformar a grande biodiversidade Amazônica em savana (Li et al., 2006).

Além disso, as secas atingem o fornecimento de água nas cidades, causam impactos na agricultura, colocando em risco a segurança alimentar, e atuam de forma negativa no setor pecuário, no índice de incêndios florestais e consequentemente na saúde pública
(Alpino et al., 2016). Essa mudança climática também atinge a atividade florestal devido ao desequilíbrio ecológico no ecossistema, gerando para a região enormes consequências na economia (Brown et al., 2006).

Com as elevadas temperaturas, o nível de mortalidade de árvores na região cresce. Tatton (2011) afirma que, em 2005, a floresta amazônica teve uma perda de aproximadamente 2,5 milhões de $\mathrm{km}^{2}$, e em 2010, esse valor chegou a 3,5 milhões de $\mathrm{km}^{2}$, atingindo a metade da área correspondente ao bioma amazônico que possui $5,3 \mathrm{~km}^{2}$.

Segundo Fearnside (2006), a temperatura do oceano Atlântico variou de forma anormal, o que afetou a climatologia da Amazônia. De acordo com Marengo et al. (2007), o El Niño não foi o responsável pela seca na região em 2005, nesse período houve um decréscimo na umidade dos alísios do Nordeste direcionado ao sul da Amazônia e uma queda no volume de pluviosidade influenciada pela baixa movimentação vertical na região. Além do mais, no período de 60 anos o Atlântico possuirá temperaturas anormais e nesse período estava em um ponto extremo (Marengo \& Nobre, 2005).

Aragão et al. (2018) mostraram que, durante a seca de 2015, o índice de incêndios florestais foi de $36 \%$, considerado alto quando comparado aos anos anteriores, tendo em vista que as áreas afetadas com focos de calor ativos correspondiam ao dobro em relação a 2005 e 2010. Os estados do Pará e Mato Grosso lideram o ranking com maior quantidade de focos de queimadas na região, 2.398 e 2.370 focos, que representam respectivamente, $34,3 \%$ e 33,9\% da Amazônia Legal (INPE, 2019). 
Haja vista que a variação no clima afeta a economia, gera impactos sociais e causa danos ao meio ambiente, o estudo da sua importância no ecossistema como um todo é essencial para obter as previsões futuras dessas variações, avaliando suas causas e consequências (Marengo et al., 2005). Os aspectos sociais, econômicos e ambientais são caracterizados e estimulados por políticas públicas que fornecem parâmetros que desenvolvem uma região no fator sustentabilidade, para isso é importante também conhecer a influência do homem em relação aos danos ambientais, principalmente para se obter medidas de conservação ambiental (Da Silva et al., 2013).

Diante disso, o presente estudo teve como objetivo estimar os impactos ambientais e socioeconômicos referentes aos três grandes últimos eventos de seca na Amazônia (2005, 2010 e 2015), com base em análise de dados estaduais de consumo de energia elétrica e dados ambientais (meteorológicos) que englobam estes aspectos.

\section{Materiais e Métodos}

As estações de coleta de dados meteorológicos deste estudo estão localizadas na região Amazônica, conforme mostra a (Figura 1). Com um total de 41 estações meteorológicas convencionais pertencentes ao Instituto Nacional de Meteorologia (INMET), os dados não têm sincronia cronológica, pois algumas estações têm informações da década de 60 até os dias atuais enquanto outras passaram a operar apenas após a década de 80 , porém com uma gama de dados climatológicos satisfatórios para todas. A região não possui características meteorológicas e climáticas definidas por ser uma região bem variável geograficamente (Santos et al., 2014). As informações são armazenadas de forma digital acessíveis para ensino e pesquisas. As medições diárias correspondem de acordo com as normas técnicas da Organização Meteorológica Mundial e estão disponíveis no BDMEP. Na plataforma online é possível consultar as seguintes variáveis:

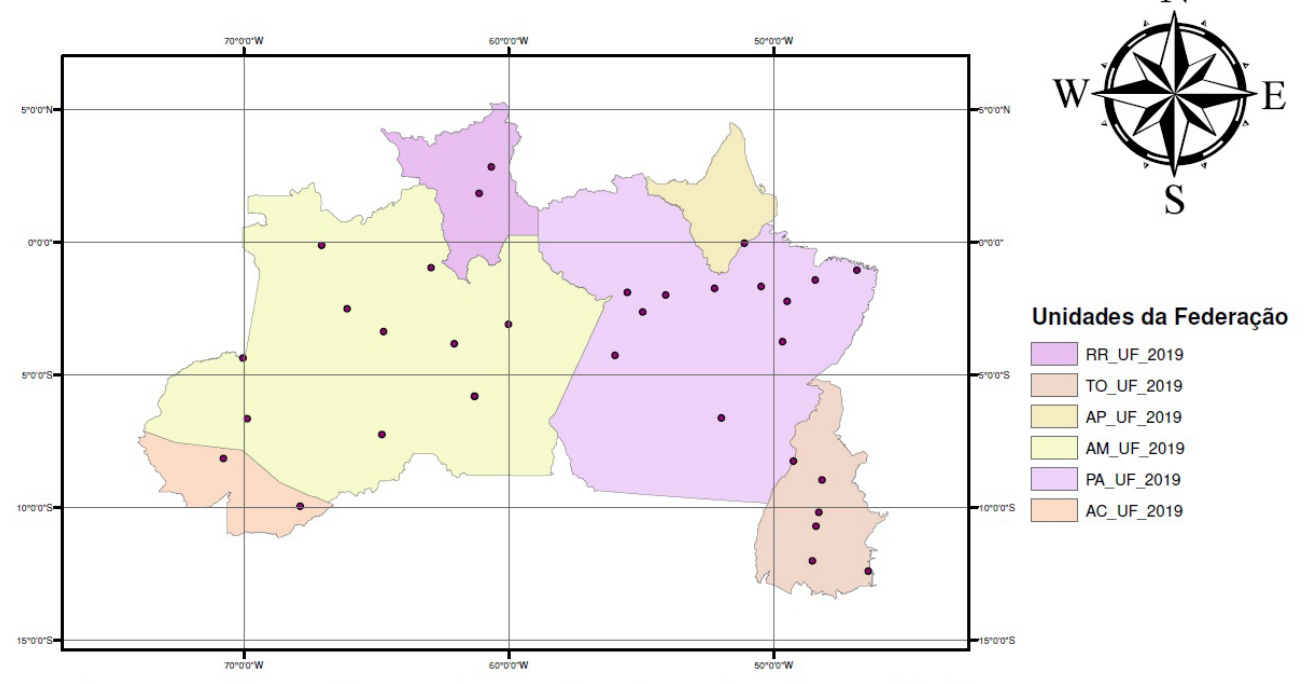

Estações Meteorológicas Convencionais Região Norte (EMC's)

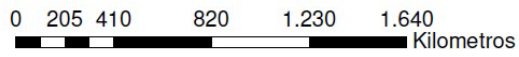

Coordinate System: GCS SIRGAS 2000 Datum: SIRGAS 2000 Units: Dearee

Figura 1 - Localização das estações meteorológicas convencionais do Instituto Nacional de Meteorologia na Amazônia.

Precipitação nas últimas 24 horas; temperatura do bulbo seco; temperatura máxima; temperatura mínima; temperatura média compensada; umidade relativa do ar; pressão atmosférica ao nível da estação; insolação; direção e velocidade do vento.
Os dados diários foram organizados no software TextPad e processados através de um script no Programa $R$ Core Team (2016) para obtenção da Normal Climatológica da Região. As normais climatológicas segundo a Organização 
Mundial Meteorológica (OMM) equivalem a valores médios de no mínimo 30 anos correspondentes as variáveis meteorológicas, durante esse período já é possível diferenciar valores climáticos padronizados de um determinado local ou região (IPMA, 2019). Portanto, nesse trabalho os dados são referentes ao período de 1978 até 2016, com um total de 38 anos.

\section{Risco de ocorrência de incêndios e consumo de energia elétrica}

Os dados de precipitação e umidade relativa foram processados em script na linguagem $R$ onde foram estimados os riscos de incêndios florestais com a aplicação da Fórmula de Monte Alegre (FMA), que leva em consideração os dados diários de precipitação e umidade relativa do ar às 13 horas. No entanto, Soares (1972) afirma que atrasos com o limite de 2 horas são aceitos em algumas exceções. No presente trabalho, foram considerados as observações referentes às 15 horas por ser o horário com informações que mais representam os valores máximos de temperatura e mínimas de umidade. Álvares et al. (2014) propuseram um modo empírico de estimativa da umidade das $13 \mathrm{~h}$ usando o valor medido ás $15 \mathrm{~h}$, que foi aplicado aos dados deste trabalho.
A escolha pela FMA se deu por ser uma fórmula originalmente brasileira, que estima o índice de riscos de incêndios de acordo com o prolongamento dos dias em relação as duas variáveis meteorológicas. Dessa forma, quanto menor for a precipitação e a umidade relativa, maior será o risco de incêndio (Soares, 1972). A Fórmula de Monte Alegre (FMA) corresponde a seguinte equação:

$$
\text { FMA }=\sum_{n-1}^{n} \frac{100}{H} \sum_{n-1}^{n} \frac{100}{H}
$$

em que $\mathrm{H}$ é a Umidade Relativa do ar com medição às 13 horas, e n é a quantidade de dias sem pluviosidade. $\mathrm{O}$ índice de Monte Alegre (IMA) é acumulativo, ou seja, quanto mais longa for a sequência de dias com baixa umidade relativa $e$ com ausência de precipitações, maiores serão os riscos climáticos para a ocorrência de incêndios. Dependendo da intensidade da chuva ocorrida, o índice sofre abatimentos em seus valores. Quando a chuva diária exceder $12,9 \mathrm{~mm}$ o IMA volta a zero, e o risco de incêndio florestal é nulo (Casavecchia, 2011). A quantidade diária de precipitação reduz o valor do índice de acordo com uma tabela de restrições (Soares 1972b). (Tabela 1).

Tabela 1 - Restrições ao somatório do IMA de acordo com a precipitação pluviométrica diária.

\begin{tabular}{|c|c|}
\hline $\begin{array}{l}\text { Precipitação diária } \\
\text { (mm) }\end{array}$ & Modificação do cálculo \\
\hline$<2,4$ & Nenhuma \\
\hline 2,5 a 4,9 & Abater $30 \%$ na FMA calculada na véspera e somar (100/H do dia) \\
\hline 5,0 a 9,9 & Abater $60 \%$ na FMA calculada na véspera e somar (100/H do dia) \\
\hline 12,0 a 12,9 & Abater $80 \%$ na FMA calculada na véspera e somar (100/H do dia) \\
\hline$>12,9$ & $\begin{array}{l}\text { Interromper o cálculo }(\mathrm{FMA}=0) \text {, recomeçando a somatória no dia seguinte ou quando a } \\
\text { chuva cessar }\end{array}$ \\
\hline
\end{tabular}

Fonte: Nunes (2005). 
Para de verificar o grau de periculosidade de incêndio, os valores de IMA foram distribuídos em classes (Tabela 2), conforme as propostas de Soares (1972).

Tabela 2 - Nível de periculosidade de ocorrência de incêndios pela FMA.

\begin{tabular}{|c|c|}
\hline Valor da Fórmula de Monte Alegre & Grau de Periculosidade \\
\hline FMA $\leq 1,0$ & NULO \\
\hline $1,1 \geq$ FMA $\leq 3,0$ & PEQUENO \\
\hline $3,1 \geq$ FMA $\leq 8,0$ & MÉDIO \\
\hline $8,1 \geq$ FMA $\leq 20,0$ & ALTO \\
\hline FMA $\leq 20,0$ & MUITO ALTO \\
\hline
\end{tabular}

Fonte: Soares (1972).

Já com base nos dados disponibilizados pela Associação Brasileira de Distribuição de Energia Elétrica (ABRADEE), observados a partir do site <http://www.abradee.org.br> foram gerados gráficos em relação ao consumo de energia elétrica e valores monetários de consumo no período de 2001 a 2016.

\section{Resultados e Discussão Variáveis ambientais}

As médias diárias de temperatura compensada média em cidades representativas dos estados do Pará (Altamira, Figura 2A), Roraima (Boa Vista, Figura 2B), Amapá (Macapá, Figura 2C) Amazonas (Manaus, Figura 2D), Acre (Rio Branco, Figura 2E) e Tocantins (Taguatinga, Figura 2F). Optou-se por escolher 1 cidade de cada estado (a exceção de Rondônia, onde não foi possível obter dados e não há disponível no BDMEP) devido ao grande número de estações, contudo tais efeitos aqui mostrados também foram detectados nas demais estações do estudo.

Os resultados mostram, em grande parte das estações analisadas, uma tendência de elevação das temperaturas ao longo do tempo. Os dados em coloração vermelha indicam os máximos de temperatura da série, enquanto a coloração verde denota as menores temperaturas.
A barra azul é uma média móvel, que permite observar a tendência dentro de períodos da série. Todas as estações mostram médias com máximos que ultrapassam os $32^{\circ} \mathrm{C}$, principalmente a partir dos anos 2000. As cidades que estão expostas ao fenômeno da friagem (Rio Branco, Manaus, Taguatinga) apresentam mínimas abaixo dos $22^{\circ} \mathrm{C}$, e seus padrões aqui mostrados corroboram o encontrado na literatura (Gomes et al., 2018; Duarte, 2006). Eventos de seca ocasionados por fenômenos de baixa frequência (El Niño Oscilação Sul) ou por alterações na TSM do Atlântico podem ocasionar a diminuição da precipitação e elevação das temperaturas em diferentes áreas da região Amazônica (Gomes et al., 2018), quanto as estações analisadas, a seca de 2005 foi observada com impactos na temperatura do ar nas estações de Altamira/PA, Manaus/AM e Rio Branco/AC, evidenciado por serem os máximos de temperatura das séries até o ano em questão. As secas de 2010 e 2015, que tem em comum sua gênese em ser um impacto do fenômeno El Niño, mostraram impactos em todas as estações estudadas evidenciado pelos máximos de temperatura do ar ocorridos nestes anos, com exceção de Taguatinga/TO, que mostrou máximos superiores em outros anos que não os mencionados, indicando que o fenômeno ENOS pode ter extensão que abrange quase toda a totalidade da região Norte Brasileira. 

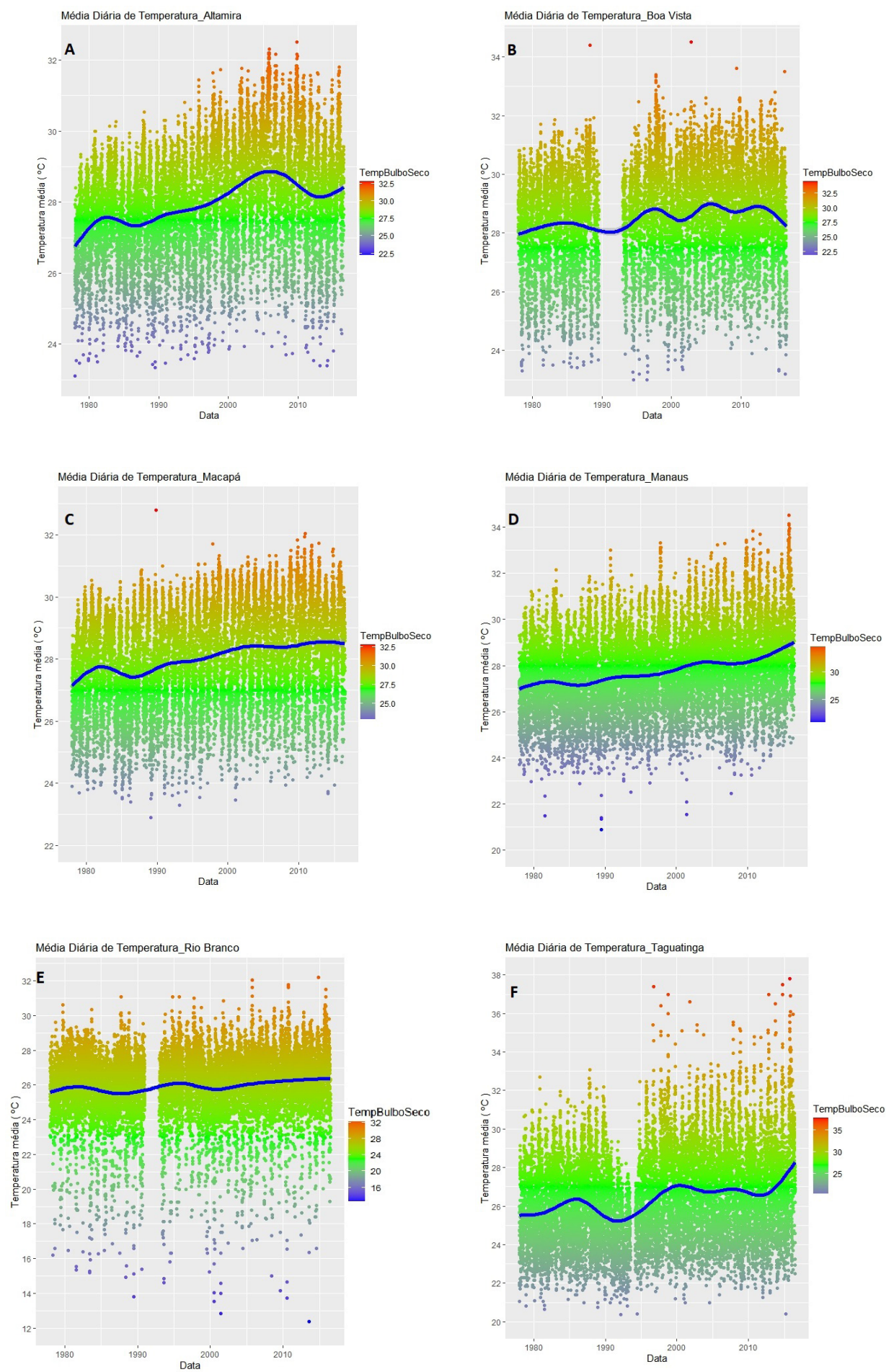

Figura 2 - Médias diárias da série histórica de temperatura do ar para as cidades de: A) Altamira/PA; B) Boa Vista/RR; C) Macapá/AP; D) Manaus/AM; E) Rio Branco/AC; e F) Taguatinga/TO.

\section{Consumo de energia elétrica}

É possível observar a variabilidade interanual do número de consumidores de energia na modalidade residencial, industrial, comercial e rural para os estados em análise Pará (Figura 3A), Amazonas (Figura 3B), Acre
(Figura 3C), Roraima (Figura 3D), Amapá (Figura 3E) e Tocantins (Figura 3F). Também se analisou a variabilidade interanual da taxa de consumo de energia elétrica (consumo/ $\mathrm{n}^{\circ}$ de consumidores) de energia residencial, industrial, comercial e rural para os estados 
do Pará (Figura 4A), Amazonas (Figura 4B), Acre (Figura 4C), Roraima (Figura 4D), Amapá (Figura 4E) e Tocantins (Figura 4F). A última e principal análise quanto ao consumo de energia elétrica nos anos é a da variabilidade interanual do consumo de energia elétrica residencial, industrial, comercial e rural para os para os estados do Pará (Figura 5A), Amazonas (Figura 5B), Acre (Figura 5C), Roraima (Figura 5D), Amapá (Figura 5E) e Tocantins (Figura 5F).

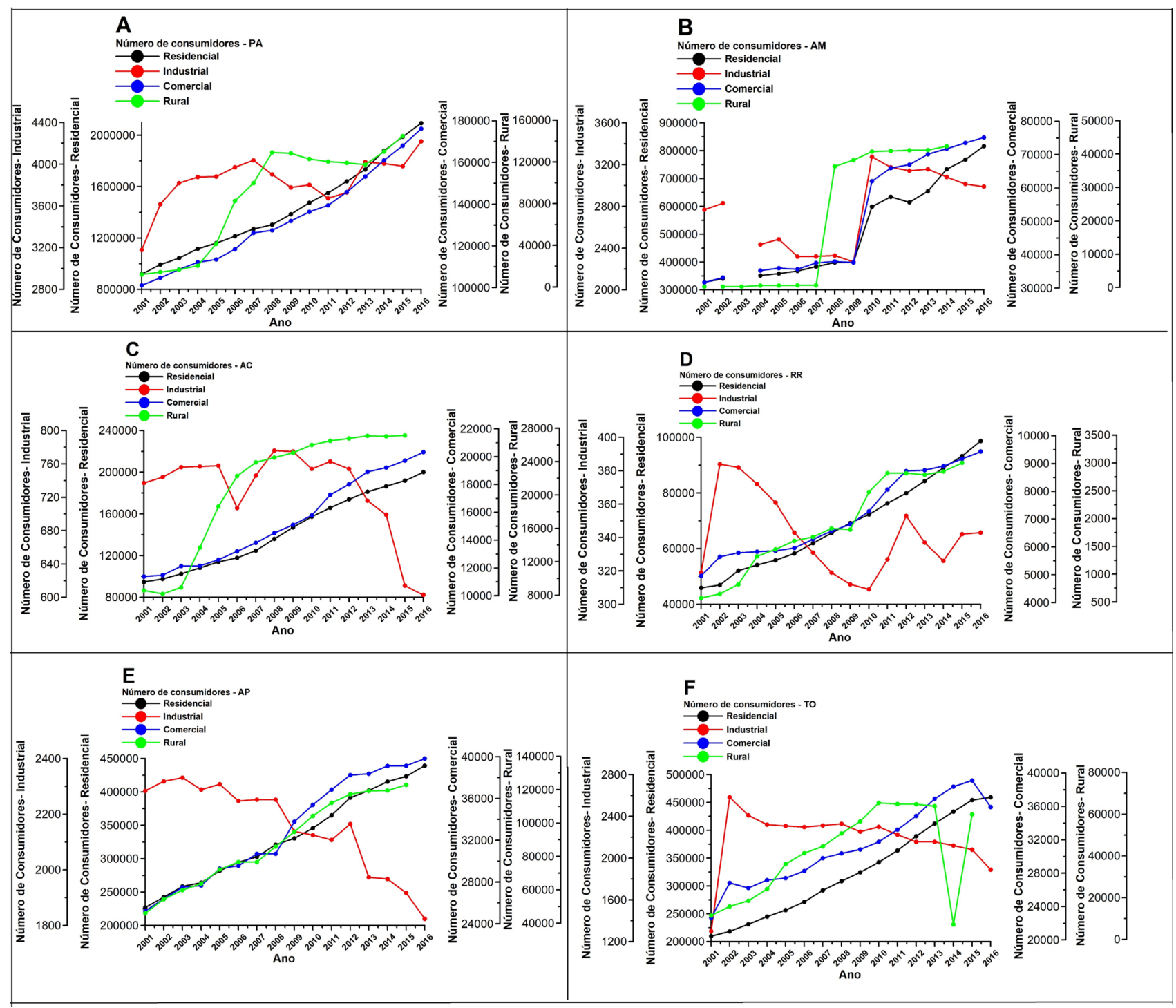

Figura 3 - Variabilidade interanual do número de consumidores de energia nos modos residencial, industrial, comercial e rural para os estados: A) Pará; B) Amazonas; C) Acre; D) Roraima; E) Amapá; e F) Tocantins. 


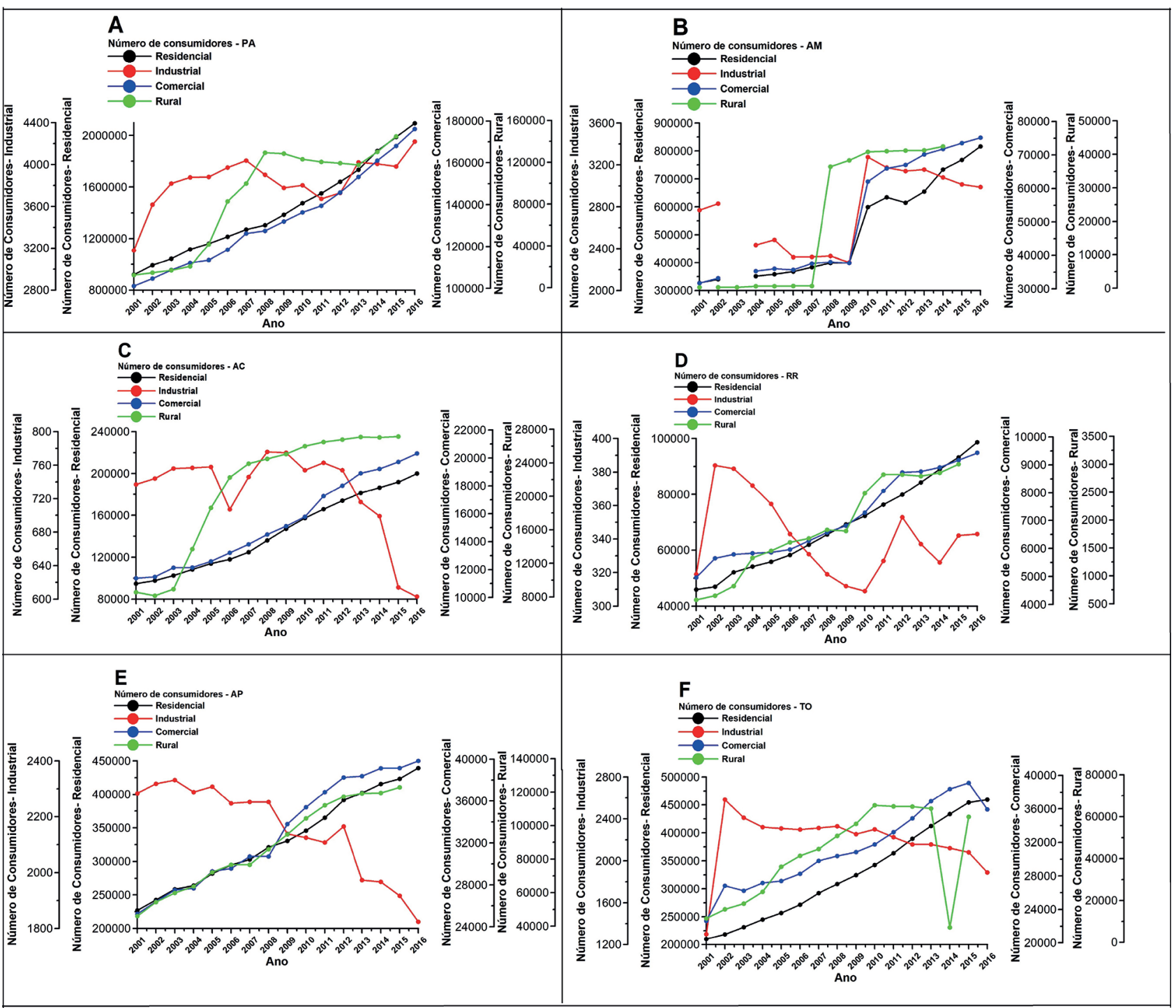

Figura 4 - Variabilidade interanual da taxa de consumo de energia elétrica (consumo $/ \mathrm{n}^{\circ}$ de consumidores) nos modos residencial, industrial, comercial e rural para os estados: A) Pará; B) Amazonas; C) Acre; D) Roraima; E) Amapá; e F) Tocantins. 


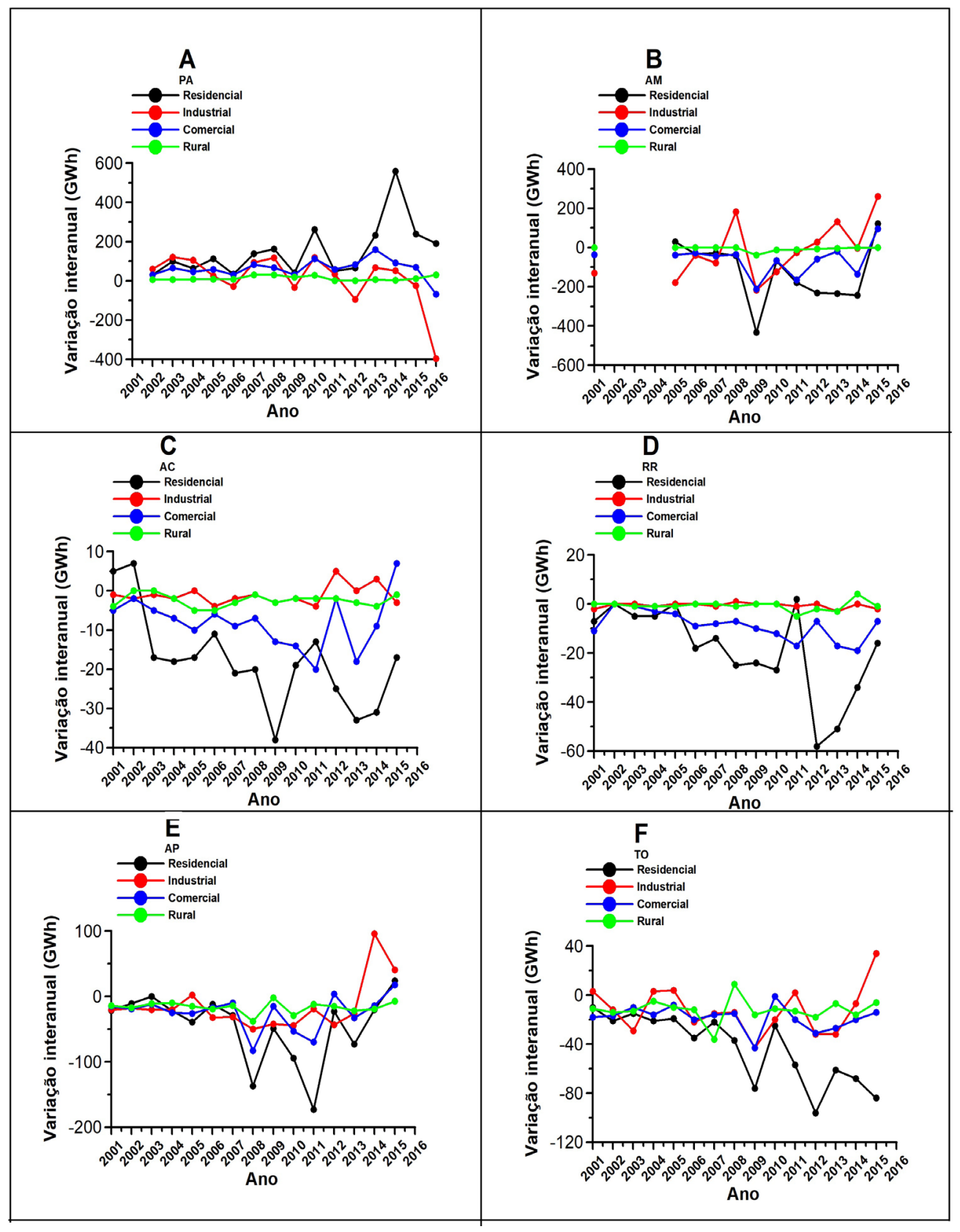

Figura 5 - Variabilidade interanual do consumo de energia elétrica (em GW/h) de energia residencial, industrial, comercial e rural para os estados: A) Pará; B) Amazonas; C) Acre; D) Roraima; E) Amapá; e F) Tocantins. 
Tais medidas comparativas se fazem necessárias para se ter noção se a variabilidade do consumo de energia elétrica entre os anos se deu por uma simples questão de aumento ou diminuição da oferta/demanda de energia pelo estado, do aumento/diminuição no número de consumidores ou por fatores de impacto ambiental que tenha ocasionado o aumento no consumo. Os dados mostram tendência de aumento contínuo nos modos de uso residência e comercial, enquanto os demais modos (industrial e rural) de uso possuem variabilidade que pode depender do nível de aquecimento da economia ou da adoção de políticas públicas quanto a expansão da oferta de energia para áreas remotas dos grandes centros. Espera-se que o consumo total de energia no setor residencial seja crescente (EPE, 2016), resultado conjunto do aumento da renda médias das famílias, do número de novos domicílios, das políticas de eficiência energética e da expansão da malha de distribuição de combustíveis, portanto a variabilidade interanual no consumo destas modalidades não pode ser atribuída a nenhum outro fator que não seja o aumento ou diminuição do uso de eletrodomésticos e eletroeletrônicos. Apesar de todos os estados mostrarem um aumento no número de consumidores (com foco no consumo residencial, curva preta), praticamente linear com o tempo, a taxa de consumo/número de consumidores sofre variação interanual (linha roxa), mostrando que o consumo de energia elétrica dos estados não se explica somente pelo aumento da demanda, e podem ser facilmente correlacionadas com eventos que instiguem o maior uso de equipamento eletroeletrônicos, como a realização de jogos esportivos de grande atratividade midiática (olímpiadas, copa do mundo) que podem induzir um maior uso de aparelhos televisores e outros eletrodomésticos nas residências, bares e outros locais durante o período das competições, até fatores climáticos como secas prolongadas, que pode induzir a população a usar mais (e por mais tempo) aparelhos de refrigeração, como condicionadores de ar e ventiladores. Excluindo anos de realização de grandes eventos esportivos no Brasil (copa do mundo de futebol, em 2014), os anos de 2010 e 2015 (secas ocasionadas por El Niño, Gomes et al., 2018) mostraram picos de máximo consumo na série de todos os estados, principalmente no modo de consumo residencial de energia elétrica. $\mathrm{AC}, \mathrm{RR}$ e PA foram os estados que mostraram picos no ano de 2005, seca ocasionada por variações da
TSM do Atlântico (Gomes et al., 2018; Fearnside, 2006).

\section{Risco de incêndios}

As médias diárias do índice de Monte Alegre (IMA) calculado pela FMA em cidades representativas dos estados do Pará (Altamira, Figura 6A), Roraima (Boa Vista, Figura 6B), Amapá (Macapá, Figura 6C) Amazonas (Manaus, Figura 6D), Acre (Rio Branco, Figura 6E) e Tocantins (Taguatinga, Figura 6F) são mostradas a seguir. Optou-se por escolher 1 cidade de cada estado là exceção de Rondônia, onde não foi possível obter dados e não há disponível no BDMEP) devido ao grande número de estações, contudo tais efeitos aqui mostrados também foram detectados nas demais estações do estudo.

Os resultados mostram uma grande sazonalidade do risco de ocorrência de incêndios na maioria das cidades analisadas, com valores próximos de 0 (risco de incêndio nulo) no período chuvoso até valores extremamente altos (acima de 800 em Taguatinga) e acima do limite para considerar muito alto o risco de incêndio $(>=20)$. Cidades localizadas em regiões com totais pluviométricos e umidade relativa muito alta ao longo do ano todo (no Amazonas, por exemplo) possuem os menores valores máximos da série, não ultrapassando um IMA de 200. Já cidades localizadas em regiões com uma estação seca bem definida e totais pluviométricos e índices de umidade relativa menores (Taguatinga, por exemplo), podem atingir FMA acumulado até acima de 800 , principalmente devido à umidade relativa muito baixa (em torno de 20\%) e a grande sequência de dias sem chuva nos locais. Estes resultados são similares aos encontrados na literatura utilizando a mesma metodologia e para cidades em localidades próximas ou dentro da área analisada neste trabalho (Silva et al., 2020; Soriano et al., 2015; Ribeiro et al., 2011).

Eventos meteorológicos extremos de baixa frequência (fenômeno ENOS - El Niño Oscilação Sul) históricos são facilmente identificados na maioria das séries devido aos picos de IMA acima da média dos máximos dos outros anos, como os eventos de 90/93, 97/98 (considerados de intensidade forte) e os eventos foco deste estudo, como a seca de 2005 (em Altamira, Macapá e Rio Branco) e as secas de 2010 e 2015 (Altamira, Rio Branco, Manaus, Macapá). Estes dados podem 
servir de subsídio para a adoção de protocolos e políticas públicas de prevenção/combate aos incêndios florestais nas áreas analisadas, dado o elevado risco de ocorrência de incêndios que as condições climáticas em anos de secas mostram neste estudo, partindo do ponto que estas são hoje previsíveis em diversos modelos climáticos (Silva et al., 2010).

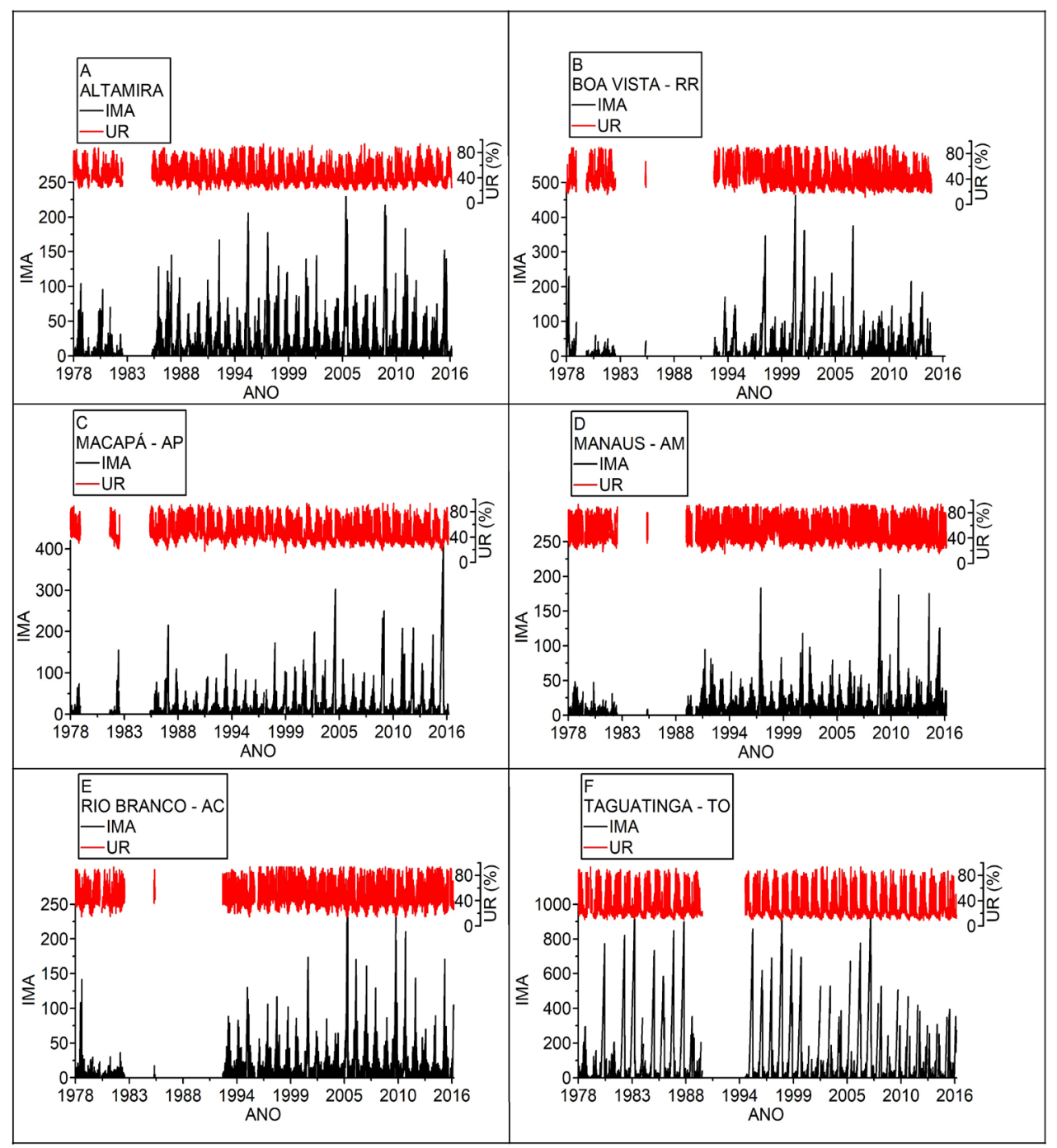

Figura 6 - Série histórica dos dados de umidade relativa do ar (às 15h) e a Fórmula de Monte Alegre acumulado diário para a cidade de Altamira.

\section{Conclusão}

As secas de 2005, 2010 e 2015, embora tenham gêneses diferentes $(2005$ devido às anomalias no atlântico, 2010 e 2015 devido aos fortes El Niños) mostraram fortes impactos ambientais (médias diárias de temperatura do ar acima de $32^{\circ} \mathrm{C}$, valores de umidade relativa do ar próximos de $20 \%$, fator de risco de ocorrência de incêndios florestais mais que o dobro de anos normais) e socioeconômicos (aumento no consumo de energia elétrica em até 200GWh nos anos de seca, principalmente no modo residencial), indicando que anos com previsão de ocorrência de secas severas como os estudados neste trabalho precisam do preparo do poder público quanto os efeitos da elevação das temperaturas, como o aumento no risco de incêndios florestais e aumento do consumo energético, necessitando medidas profiláticas quanto os impactos negativos gerados. 


\section{Agradecimentos}

$\mathrm{O}$ primeiro autor agradece à PROPPITUFOPA pela concessão de bolsa de iniciação científica durante a realização do trabalho. Os autores agradecem aos avaliadores pelas valiosas contribuições na melhoria do artigo.

\section{Referências}

Alvares CA, Cegatta IR, Vieira LAA, Pavani RDF, Mattos EM, Sentelhas PC, Stape JL \& Soares RV. Perigo de Incêndio Florestal: Aplicação da Fórmula de Monte Alegre e Avaliação do Histórico para Piracicaba/Sp. Scientia Forestalis, Piracicaba 42(104): 511-22, 2014.

Ambrizzi $\mathrm{T}$ et al. Cenários regionalizados de clima no Brasil para o século XXI: Projeções de clima usando três modelos regionais. Relatório 3. Ministério do Meio Ambiente (MMA), Secretaria de Biodiversidade e Florestas (SBF), Diretoria de Conservação da Biodiversidade (DCBio). Mudanças Climáticas Globais e Efeitos sobre a Biodiversidade - Subprojeto: Caracterização do clima atual e definição das alterações climáticas para o território brasileiro ao longo do século XXI. Brasília, fevereiro de 2007.

ABRADEE (Associação Brasileira de Distribuição de Energia Elétrica). Segmento de Distribuição, 2018. Disponível em: http://www.abraade.org.br/ setor-de-distribuição/banco-de-dados/. Acessado em 15/06/2018.

Alpino TA, Sena MRA \& Freitas MC. Desastres Relacionados a seca e a saúde coletiva. Revista Ciência e Saúde Coletiva 21(3): 809-820. Rio de Janeiro, 2016.

Aragão LEOC, Anderson LO, Fonseca MG, Rosan TM, Vedovato LB, Wagner FH et al. (2018). 21st Century drought-related fires counteract the decline of Amazon deforestation carbon emissions. Nat. Commun. 9: 536. doi: 10.1038/s41467-017-02771-y

Brown IF, Schroeder W, Setzer A, Maldonado M, Pantoja N, Duarte A \& Marengo J. Fires in rainforests of south western Amazonia: Multi-national satellite imagery for monitoring and for informing the public. EOS Transactions, 87(26): 253-264, 2006.

Casavecchia BH, Souza AP \& Stangerlin DM. Análise dos riscos de ocorrência de incêndios florestais no município de Sinop/MT. In: Congresso Brasileiro de Agrometeorologia, 2011, Guaraparí/ES. Anais do $17^{\circ}$ Congresso Brasileiro de Agrometeorologia. Vitória/ES: DCM/Incaper, 2011.

Cavalcanti IFA \& Kousky V. Drought in Brazil During Summer and Fall 2001. Associated Atmospheric Circulation Feature, Climanalise, 2(1): 110. 2004.
Cox $\mathrm{P}$ et al. Increase risk of Amazonian Drought dueto decreasing aerossol pollution. Nature, 453: 212-216. 2008.

Da Silva VPR, Aleixo DO, Neto JD, Maracajá KFB \& De Araújo LE. Uma medida de sustentabilidade ambiental: Pegada Hídrica. Revista brasileira de Engenharia Agrícola e Ambiental, 17(1): 100-105, 2013.

Duarte AF. Aspectos da climatologia do Acre, Brasil, com base no intervalo 1971 - 2000. Revista Brasileira de Meteorologia, 21: 308-317, 2006.

EPE (Empresa de Pesquisa Energética). 2016. Plano decenal de expansão de energia.

Fearnside PM \& Graça PMLA. BR-319: Brazil's Manaus - Porto Velho. High wayand the potential impacto flinking the arc of deforestation to central Amazonia. Environmental Management. 38(5): 705-716. 2006.

Galbraith D, Levy PE, Sitch S, Huntingford C, Williams M \& Meir P. Multiple mechanisms of Amazonian forest biomass losses in three dynamic global vegetation model sunder climate change. New Phytologist, 2010.

Gomes ACS, Costa GB, Silva JT, Coutinho MDL, Costa MS \& Fitzjarrald DR. Seca e a saúde das populações residentes em regióes da Amazônia Brasileira nos anos de 2005, 2010 e 2015. Revista Brasileira de Climatologia, 23: 289-306, 2018.

Good P, Jones C, Lowe J, Betts R, Booth B \& Huntingford C. Quantifying Environmental Drivers of Future Tropical Forest Extent. Journal of Climate, 2011.

Hegerl GC, Crowley J, Hyde WT \& Frame DJ. Climates sensitivity constrained by temperature reconstructions over the past seven centuries. Nature, 440: 1029-1032. 2006.

Hogan DJ. Demographic aspects of global environmental change: What is Brazil's contribution? p: 15-41. In: Hogan DJ \& Tolmasquim MT (eds.), Human Dimensions of Global Environmental Change. Rio de Janeiro, Academia Brasileira de Ciências, 2001.

IBGE (Instituto Brasileiro de Geografia e Estatística). 2018. Estatística/Download. Disponível em: <http:// www.ibge.gov.br>. Acesso em: 13 de junho de 2018.

INMET (Instituto Nacional de Meteorologia). 2018. Dados da Rede do INMET. Disponível em: http://www. inmet.gov.br. Acesso em: 15 de junho de 2018.

IBGE (Instituto Brasileiro de Geografia e Estatística). 2014. Bioma Amazônia, 2014. Disponível em <http:// www.ibge.gov.br>.

INPE (Instituto Nacional de Pesquisas Espaciais). 2019. BD Queimadas. Disponível em http://www.inpe.br/ queimadas/portal. Acesso em 02 de setembro de 2019. 
INPE (Instituto Nacional de Pesquisas Espaciais). 2003. Monitoramento da Floresta Amazônica por satélite 2000, 2001, 2002 e 2003. Separata. São José dos Campos, 2003.

IPMA (Instituto Português do Mar e da Atmosfera). 2019. Normais Climatológicas. Disponível em http:// www.ipma.pt. Acesso em: 08 abr. 2019.

IPCC (Intergovernamental Panel on Climate Change). 2001. Third Assessment Report: Climate Change 2000. Intergovernmental Panel on Climate Change. Cambridge Univ. Press.

IPCC (Intergovernamental Panel on Climate Change). 2018. Summary for Policymakers. In: Global warming of $1.5^{\circ} \mathrm{C}$. An IPCC Special Report on the impacts of global warming of $1.5^{\circ} \mathrm{C}$ above pre - industrial levels and related global greenhouse gas emission pathways, in the context of strengthening the global response to the threat of climate change, sustainable development, and efforts to eradicate poverty [Masson-Delmotte V, Zhai P, Pörtner HO, Roberts D, Skea J, Shukla PR, Pirani A, Moufouma-Okia W, Péan C, Pidcock R, Connors S, Matthews JBR, Chen Y, Zhou X, Gomis MI, Lonnoy E, Maycock T, Tignor M \& Waterfield T (eds.)]. World Meteorological Organization, Geneva, Switzerland, 32 p.

Jon P. Agricultural Development Under a Changing Climate: Opportunitie sand Challenges for Adaptation. Joint Departmental Discussion. Agriculture and Rural Development \& Environment Departments. The World Bank, p. 1, 2009.

Li W, Fu R \& Dickinson RE. "Rainfall and its seasonality over the Amazon in the 21st century as assessed by the coupled models for the IPCC AR4". Journal of Geophysical Research: Atmospheres, 111: D02, 2006.

Luiz EOC et al. 21st Century drought-related fires counteract the decline of Amazon deforestation carbon emissions. Nature Communications. 2018.

Marengo JA et al. The drought of Amazonia in 2005. Journal of Climate. 21: 495-516. 2008a.

Marengo JA, Nobre C, Tomasella J, Cardoso M \& Oyama M. Hydroclimatic and ecological behaviour of the drought of Amazonia in 2005. Philosophical Transactions of the Royal Society of London. Biological Sciences, 21: 1-6, 2008b.

Marengo JA \& Nobre C. Tempo e clima no Brasil. Cap. 13, clima da região Amazônica. 2005.

Marengo JA et al. Eventos extremos em cenários regionalizados de clima no Brasil e América do Sul para o Século XXI: Projeções de clima futuro usando três modelos regionais. Relatório 5, Ministério do Meio Ambiente (MMA), Secretaria de Biodiversidade e Florestas (SBF), Diretoria de Conservação da
Biodiversidade (DCBio). Mudanças Climáticas Globais e Efeitos sobre a Biodiversidade - Subprojeto: Caracterização do clima atual e definição das alterações climáticas para o território brasileiro ao longo do século XXI. Brasília, fevereiro de 2007.

Marengo JA. Mudanças climáticas globais e seus efeitos sobre a biodiversidade - caracterização do clima atual e definição das alterações climáticas para o território brasileiro ao longo do século XXI. 2. ed. Brasília: Ministério do Meio Ambiente, 1: 214, 2007.

Marengo JA et al. "The Drought of Amazonia in 2005". Journal of Climate. 21(3), 2007.

Marengo JA. On the hydrological cycle of the Amazon Basin: A historical review and currentstate - of theart. Revista Brasileira de Meteorologia, 21(3): 1-19, 2006.

Marengo JA. Impactos Extremos relacionados com o tempo e o clima - Impactos sociais e econômicos. Boletim do Grupo de Pesquisa em Mudanças Climáticas - GPMC. Instituto Nacional de Pesquisas Espaciais (INPE). Centro de Sistema do Sistema Terrrestre. São Paulo, Brasil, Maio de 2009.

Marengo JA et al. Development of regional future climate changes cenarios in South America using the Eta CPTEC/HadCM3 climate change projections: climatology and regional analysis for the Amazon, São Francisco and the Paraná River basins. Climate Dynamics, 38: 1829-1848, 2012.

Marengo JA \& Nobre C. Lições do Catarina e do Katrina. As mudanças do clima e os fenômenos extremos. Ciência Hoje. 37(221): 22-27. 2005.

Nobre C \& Assad ED. Mudança ambiental no Brasil. Em Terra na estufa, edição especial Scientific American Brasil, (12): 70-75. 2005.

Nunes JRS. FmA+ - um novo índice de perigo de incêndios florestais para o Estado do Paraná - Brasil. 2005. 150 f. Tese (Doutorado Engenharia Florestal), UFPR, Curitiba, 2005.

Oyama MD \& Nobre CA. A new climate-vegetation equilibrium state for Tropical South America. Geophysical Research Letters 30(23): 2199-2203. 2003.

Parry ML, Canziani OF, Palutikof JP, Van Der Linden PJ \& Hanson CE. Climate change 2007: Impacts, adaptation and vulnerability. Contribution of Working Group II to the Fourth Assessment Report of the Intergovernmental Painel on Climate Change, Cambridge University Press, Cambridge, UK, 982 p. 2007.

Pereira MPS. Interação entre ecossistemas terrestres e a atmosfera na Amazônia: conexões biogeofisicas e 
biogeoquimicas. Dissertação de mestrado. Universidade federal de viçosa. Viçosa. Minas gerais. 2006.

R CORE TEAM. R: A language and environment for statistic al computing. R Foundation for Statistic al Computing, Vienna, Austria.URL https://www.Rproject.org/. 2016.

Salazar LF, Nobre CA \& Oyama MD. Climatic change consequences on the biome distribution in tropical South America. Geophysical Research Letters, 34: L09708. 2007.

Ribeiro L, Soares RV, Batista AC \& Silva IC. Análise do perigo de incêndios florestais em um município da Amazônia Mato-grossense, Brasil. Floresta, 41(2): 257270. doi: http://dx.doi.org/10.5380/rf.v41i2.21873. 2011.

Salati E \& Marques J. 1984. Climatology of the Amazon region. In: Sioli $\mathrm{H}$ (ed). The Amazon - Limnology and landscape ecology of a mighty tropical river and its basin. Dr. W. Junk Publishers, Bonn, Alemanha, p. 85126, 1984. 2014. Precipitation regionalization of the Brazilian Amazon. Atmospheric Science Letters. DOI: 10.1002/asl2.535.2014.

Santos EB, Lucio OS, Santos E \& Silva CM. Precipitation regionalization of the Brazilian Amazon. Atmospheric Science Letters. DOI: 10.1002/asl2.535. 2014.

Serrão E, Santos C, Wanzeler R, Gonçalves L \& Lima A. Avaliação da seca na Amazônia: Análise da Bacia Hidrográfica do Rio Solimões. Rev. Geogr. Acadêmica 9(2). 2015.

Settele J, Scholes R, Betts R, Bunn S, Leadley P, Nepstad D et al. Terrestri alandinl and water systems. In: Field CB, Barros VR, Dokken DJ, Mach KJ, Mastrandrea MD, Bilir TE et al. (ed.). Climate Change 2014: Impacts, Adaptation, and Vulnerability. Part A: Global and Sectoral Aspects. Cambridge University Pres. s, Cambridge, United Kingdomand New York, NY, USA, p. 271-359. 2014.
Silva BB, Ferreira MAF, Silva VPR \& Ferreira RC. Desempenho de modelo climático aplicado à precipitação pluviométrica do Estado de Pernambuco. Revista Brasileira de Engenharia Agrícola e Ambiental, 14. 2010.

Silva IDB, Valle ME, Barros LC \& Meyer JFCA. A wildfire warning system applied to the state of Acre in the Brazilian Amazon, Applied Soft Computing, Volume 89, 2020.

Silvério DS, Silva AA \& Moutinho P. Instituto de Pesquisa Ambiental na Amazônia - IPAM. Nota Técnica: Amazônia em chamas. Brasília/DF, agosto de 2019.

Soares RV. Determinação de um índice de perigo de incêndio para a região centro-paranaense, Brasil. 1972. 72 f. Dissertação (Mestrado em Ciências) Centro Tropical de Ensino e Investigação, Instituto Interamericano de Ciências Agrícolas OEA, Turrialba, Costa Rica, 1972.

Soriano BMA, Daniel O \& Santos SA. Eficiência de índices de risco de incêndios para o pantanal SulMato-Grossense. Ciência Florestal, Santa Maria, 25(4): 809-816, 2015.

Stainforth DA et al. Uncertainty in predictions of the climate response torising levels of greenhouse gases. Nature 433: 403-406, 2005.

Tatton JEG. Água e Mudanças Climáticas. Cidadania e Meio Ambiente, (32): 22-24, 2011.

Zeng N, Yoon JH, Marengo JA, Subrmanaiam A, Nobre CA, Mariotti A \& Nellin JD. Causes and impacts of the Amazon drought. Environmental Research. 3: 1-6, 2008.

Biodiversidade Brasileira - BioBrasil.

Edição Temática: Análise de Componentes do Sistema Climático e a Biodiversidade no Brasil

$$
\text { n. 4, } 2021
$$

http://www.icmbio.gov.br/revistaeletronica/index.php/BioBR

Biodiversidade Brasileira é uma publicação eletrônica científica do Instituto Chico Mendes de

Conservação da Biodiversidade (ICMBio) que tem como objetivo fomentar a discussão e a disseminação de experiências em conservação e manejo, com foco em unidades de conservação e espécies ameaçadas.

ISSN: 2236-2886 